\title{
Gestión de la innovación en las instituciones de educación superior*
}

[Notas metodológicas y de investigación]

\author{
Evelyn Garnica Estrada* \\ José Alejandro Franco Calderón $n^{* * *}$
}

Recibido: 1 de septiembre de 2020

Revisado: 10 de septiembre de 2020

Aceptado: 11 de septiembre de 2020

Cómo citar este artículo:

Garnica Estrada, E. y Franco Calderón, J. A. (2020). Gestión de la innovación en las instituciones de educación superior. Signos, Investigación en sistemas de gestión, 13(1). https://doi.org/10.15332/24631140.6338

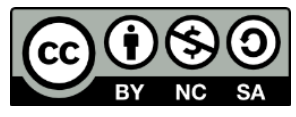

\footnotetext{
* Nota metodológica

** Corporación Universitaria Republicana. Doctora en Educación. Magíster en Dirección de Proyectos. Especialista en Planeación, Desarrollo y Administración de la Investigación. Ingeniera de Diseño y Automatización Electrónica. Docente investigadora del Grupo de Investigación Desarrollo e Innovación Sostenible (GIDIS) de la Corporación Universitaria Republicana. Bogotá, Colombia. Correo electrónico: egarnicae@urepublicana.edu.co, evelyngarnica@gmail.com. ORCID: 0000-0002-62057817. CVLAC:

https://scienti.minciencias.gov.co/cvlac/visualizador/generarCurriculoCv.do?cod_rh $=0$ $\underline{001142844}$

${ }_{* * *}$ Corporación Universitaria Republicana. Magíster en Ingeniería Electrónica. Especialista en Diseño de Aplicaciones para Televisión Digital Interactiva y en Administración de Tecnologías de la Información para la Comunicación Virtual. Ingeniero electrónico. Docente investigador del Grupo de Investigación Desarrollo e Innovación Sostenible (GIDIS) de la Corporación Universitaria Republicana. Bogotá, Colombia. Correo electrónico: alejing@urepublicana.edu.co, alejing@gmail.com. ORCID: 0000-0003-3931-2186. CVLAC:

https://scienti.minciencias.gov.co/cvlac/visualizador/generarCurriculoCv.do?cod_rh $=0$ $\underline{001410032}$
} 


\section{Resumen}

La innovación en las instituciones de educación superior hace parte de su quehacer científico, tecnológico y humanista. Es un proceso dinámico y transformador que, al ser gestionado con una estructura definida, sirve de apoyo a las iniciativas académicas y de investigación, fomenta la generación y gestión de conocimiento y, en esta vía, aporta al sistema nacional de ciencia, tecnología e innovación. El presente artículo presenta tres procesos generales (directivo, académico y administrativo) que deben coexistir para que la innovación sea un elemento orgánico en los procesos académicos y funcionales de las instituciones. Las innovaciones en docencia, investigación e infraestructura se alinean con las de carácter administrativo, lo que refleja una correlación entre todas las áreas de la institución y evidencia que la innovación no es unidireccional, pues se puede implementar de forma integral.

Palabras clave: gestión, innovación, investigación, conocimiento.

\section{Managing innovation in higher education institutions}

\section{Abstract}

Innovation in higher education institutions is part of their scientific, technological, and humanistic endeavors. It is a dynamic and transforming process that, when managed with a defined structure, supports academic and research initiatives, promotes knowledge generation and management and, thus, contributes to the national system of science, technology, and innovation. This article presents three general processes (managerial, academic, and administrative) that must coexist so that innovation to be an organic element in the academic and functional processes of institutions. Innovations in teaching, research and infrastructure are aligned with those of an administrative nature, reflecting a correlation between all the institution areas and shows that innovation is not unidirectional, since it can be implemented in an integrated manner. 
Keywords: Management, Innovation, Research, Knowledge.

\section{Gestão da inovação nas instituições de ensino superior}

\section{Resumo}

A inovação nas instituições de ensino superior faz parte do seu trabalho científico, tecnológico e humanístico. Trata-se de um processo dinâmico e transformador que, quando gerido com uma estrutura definida, apoia iniciativas académicas e de pesquisa, promove a geração e gestão do conhecimento e, desta forma, contribui para o sistema nacional de ciência, tecnologia e inovação. Este artigo apresenta três processos gerais (de direção, acadêmico e administrativo) que devem coexistir para que a inovação seja um elemento orgânico nos processos acadêmicos e funcionais das instituições. As inovações nos domínios do ensino, da pesquisa e das infraestruturas estão alinhadas com as de natureza administrativa, refletindo uma correlação entre todas as áreas da instituição e evidência de que a inovação não é unidirecional, uma vez que pode ser implementada de forma integrada.

Palavras-chave: gestão, inovação, pesquisa, conhecimento.

\section{Introducción}

A medida que las instituciones de educación superior se convierten gradualmente en uno de los ejes fundamentales del sistema de producción de conocimiento de la sociedad, su papel en la innovación se vuelve más diverso. Por esta razón, la gestión de la innovación se ha convertido en el motor que impulsa el desarrollo de una cultura innovadora dentro de la academia, para sensibilizar a los estudiantes y docentes en la generación de proyectos productivos de base tecnológica, generar producción de nuevo conocimiento, fomentar alianzas y redes de apoyo, articular procesos constructivos que conllevan el desarrollo de conocimiento 
tecnológico, promover la inversión en las instituciones y canalizar el potencial en los resultados generados de un proceso de innovación desde el producto hasta todos los actores que intervienen en el proceso.

Diversos estudios (Banegas, 1999; García, 1999; Angel, 2006) definen las características de una cultura de innovación, entre las cuales resaltan: mayor creatividad, educación ubicua y permanente, mosaico cultural, pasión por la investigación, desarrollo de confianza, reconocimiento social, incentivos, aceptación del riesgo, anticipación de necesidades, control, aceptación del cambio, entre otros.

En este panorama, según Peter Drucker (2002), algunas fuentes que pueden dar origen a un proceso de innovación son el éxito o fracaso inesperado; las incongruencias o disonancias; las necesidades en los procesos internos de las instituciones; los cambios en los mercados; los valores y las percepciones de la sociedad; los nuevos conocimientos, y las nuevas tecnologías.

Cualquiera que sea la fuente, la innovación puede ser definida, estructurada y organizada, es decir, gestionada (Goldenberg et ál., 2003).

De esta forma, se logran buenas prácticas de alta aplicabilidad y transferencia para la promoción una cultura de la innovación, junto con la formación académica, recursos, misión institucional, identidad y autonomía. Asimismo, se dirigen esfuerzos de participación en la construcción del conocimiento y aportes significativos a la comunidad académica y científica que impulsen el desarrollo del país.

\section{Gestión del conocimiento en procesos de innovación}

El conocimiento se considera un activo en la economía actual y, por esta razón, se ha convertido en un motor económico e indicador de productividad en el ámbito institucional. Es un recurso que se puede 
generar, transformar y transferir. En ese sentido, la llamada economía basada en el conocimiento hace que se planteen iniciativas desde el ámbito académico para promoverla e impactar en el desarrollo y la competitividad económica del país. La sociedad del conocimiento, caracterizada precisamente por el conocimiento como un recurso estratégico, es la clave para que las instituciones y los países sean más competitivos y se puedan alinear en el marco de los sectores de talla mundial y las megatendencias sociales y tecnológicas (Aponte, 2016).

Según Albornoz y López (2010), en la actualidad los países de habla hispana han ido adquiriendo mayor sensibilidad acerca del papel de la ciencia, la tecnología, el conocimiento y la innovación en el desarrollo de las naciones, por lo que se ha convertido en una cuestión de Estado objeto de políticas específicas. Para el caso de Colombia, esto se evidencia en los lineamientos dados por el Ministerio de Ciencia, Tecnología e Innovación (Minciencias), organismo rector del sector y del Sistema Nacional de Ciencia, Tecnología e Innovación (SNCTI), que tiene como objetivo fomentar la innovación en las empresas colombianas mediante el apalancamiento de fuerzas para crear un músculo que permita dar el apoyo necesario a los empresarios e innovadores, a través de instrumentos, recursos y capacidad operativa. Otra de las políticas de Minciencias es promover, fomentar y apoyar la generación de conocimiento, innovación y desarrollo tecnológico por medio de la formación permanente del recurso humano, la transferencia e intercambio de tecnologías y una continuada actividad científico-tecnológica en forma conjunta entre la academia, los sectores productivos, la innovación y el desarrollo (Ministerio de Ciencia, Tecnología e Innovación, 2020). La innovación en las instituciones de educación superior (ies) tiene como finalidad contribuir al desarrollo social, cultural y tecnológico del país, y al mejoramiento de procesos del sector académico; en algunos casos, de 
forma articulada con el sector productivo o el Gobierno mediante el aporte de recursos para elevar sus niveles de productividad. Es en este punto donde la cadena de gestión del conocimiento incide en el fortalecimiento de las capacidades de los actores involucrados, por lo que asimilar, utilizar, adaptar y cambiar son acciones clave para el desarrollo de nuevos productos y procesos, y para el mejoramiento de las condiciones de competitividad.

\section{Procesos de la gestión de la innovación}

La gestión está orientada a la acción y la solución creativa de los problemas de la administración dentro de un contexto de innovación (Ospina, 1993). La innovación es novedad, utilidad y sostenibilidad; se traduce en productividad porque mejora los resultados reduciendo de forma sustancial los tiempos que normalmente se invertían (Organización para la Cooperación y el Desarrollo y Oficina Estadística de la Unión Europea, 2007).

Según Benavides (2008), la innovación debe ser conceptual y práctica; de esta forma, se perfecciona la capacidad de investigación, desarrollo e innovación en los procesos académicos y funcionales de las ies, de manera que logren aumentar los niveles de productividad y competitividad. Deben existir tres procesos que interactúan como entradas y salidas a los procesos directivo, académico y administrativo, de acuerdo con la función de cada uno.

\section{Proceso directivo}

Se trata de la primera fase de los sistemas de gestión en términos generales, debido a que propone las bases de direccionamiento estratégico para su implementación. En ese sentido, se definen los conceptos más 
generales sobre investigación, innovación y desarrollo, además de señalar el norte de la institución en términos de innovación.

Los centros, departamentos o áreas de investigaciones de las instituciones, decanaturas, coordinaciones y vicerrectorías, así como los docentes investigadores, son parte de este proceso y están encargados de definir los lineamientos estratégicos del sistema para su implementación y desarrollo.

Este proceso directivo tiene como base el diseño de la formación académica desde los aspectos curriculares, el modelo pedagógico y el modelo institucional, y debe desarrollarse en un marco de dimensiones que promueven la investigación formativa y la innovación en las instituciones (figura 1).

Figura 1. Dimensiones que promueven la cultura de innovación

1. Actitudes y

percepciones

4. Aplicación

significativa del

conocimiento
2. Adquisición e

integración del

conocimiento

5. Desarrollo de

líneas y proyectos

de investigación
3. Refinamiento y

profundización del

pensamiento

6. Innovación,

desarrollo y

propiedad

intelectual

Fuente: elaboración propia.

La gestión de la innovación requiere una buena disposición actitudinal, confianza y buena percepción sobre las diferentes actividades y roles que se generan (De Bono, 1998). La innovación, en términos generales, es un proceso permanente de aprendizaje y de interacción con el objeto de conocimiento. Para lograr la articulación entre los procesos académicos y los de investigación, desarrollo e innovación ( $\mathrm{I}+\mathrm{D}+\mathrm{i})$, se requiere conocer las condiciones iniciales de los estudiantes, los docentes y la institución, a 
fin de tener una base de construcción y crear las oportunidades para la observación, comparación, indagación, comprensión, clarificación, predicción, verificación y comprobación (Cornejo y Muñoz, 2009). Estas acciones llevan a operaciones cognitivas que son pilares fundamentales en el proceso de innovación: comparar, clasificar, inducir, deducir, analizar errores, construir soportes para argumentar, sustentar y abstraer.

El mejoramiento de procesos, operaciones, métodos o artefactos es la razón por la cual surge la necesidad de direccionar la gestión de innovación, lo que implica tomar decisiones, experimentar, solucionar problemas reales e inventar. En los lineamientos que surgen en este proceso, los resultados apuntan hacia prototipos, nuevos modelos o métodos para gestionar cambios sustanciales bajo las líneas de conocimiento definidas en cada institución. Estas líneas definen el norte en materia de $\mathrm{I}+\mathrm{D}+\mathrm{i}$; delimitan y definen el territorio en el cual han de moverse los grupos de investigación, e inciden en los procesos de formación académica, redes, sector productivo, gestión tecnológica, innovación y productividad.

\section{Proceso académico}

Orienta la integración de la gestión de la innovación en los procesos de formación. De esta manera, la formación investigativa articula sus procesos con la formación profesional integral a través del desarrollo y fortalecimiento de competencias. En esa medida, la formulación y desarrollo de proyectos de $\mathrm{I}+\mathrm{D}+\mathrm{i}$ académicos o en conjunto con empresas genera actividades de documentación, gestión y difusión del conocimiento, articulación de expertos y promoción de la gestión del conocimiento por diferentes vías, desde la producción bibliográfica, el desarrollo de prototipos, normas y procesos hasta los resultados de propiedad 
intelectual. Todo esto tiene el propósito de retroalimentar los programas de formación académica a partir de los procesos y resultados de I+D+i.

\section{Proceso administrativo}

En esta tercera fase, se integran las acciones que apoyan la ejecución del proceso académico mediante la documentación, el posicionamiento y la visibilidad de la $\mathrm{I}+\mathrm{D}+\mathrm{i}$. En un proceso de gestión de la innovación, es fundamental direccionar los procesos administrativos. En consecuencia, con las políticas nacionales de ciencia, tecnología e innovación, junto con la producción en investigación y procesos académicos, se deben administrar los grupos en los que surgen procesos de innovación ante Minciencias. Así, todos los grupos de investigación de las instituciones deben estar registrados en GrupLAC, el sistema de información que permite actualizar la producción y avances del SNCT. GrupLAC se alimenta de CvLac, el sistema de información que almacena la información de los investigadores con el fin de atender y gestionar los índices para su respectiva clasificación y categorización.

Por otro lado, el proceso administrativo apoya la planeación de los proyectos, la asignación de los recursos, la autoevaluación y las políticas institucionales, que constituyen la base de los indicadores de gestión (proceso, resultados e impacto).

\section{De la idea a la innovación}

Verworn et ál. (2000) resaltan elementos clave de la gestión de la innovación tecnológica que permiten gestionar el proceso global, desde el inicio de la idea hasta su transformación en la innovación, en las siguientes etapas:

1. Concepción de la idea: en esta etapa se realiza un análisis de requerimientos para valorar la idea en términos de la necesidad o la 
situación problema que genera. Esto da paso a la planificación de la materialización de la idea, es decir, el proyecto.

2. Implementación: esta etapa contempla el desarrollo y la construcción de modelos, prototipos y esquemas funcionales para retroalimentar aspectos técnicos y de aplicación. Permite realizar diseños experimentales, pruebas piloto y testeos generales convenientes para la última etapa.

3. Mercado de la innovación: se caracteriza por definir el estado esperado de la idea, donde se valida la producción, se realiza el lanzamiento y se introduce el producto al mercado.

En ese sentido, el proceso de innovación tiene como origen la generación ideas, que al ser analizadas se van perfilando hasta obtener el alcance técnico y funcional para su materialización; de esta forma, se proyecta la idea como innovación (Dávila et ál., 2006). La generación de ideas puede darse bajo diferentes circunstancias; sin embargo, la creatividad es un punto de partida para concebirlas y estructurarlas orgánicamente. Ahmed et ál. (2012) definen la creatividad como el proceso por medio del cual se generan ideas nuevas y útiles orientadas hacia mejoras o avances que puedan cambiar el mundo. La creatividad es la base de un proceso continuo para generar innovación (Rodríguez, 1993) y una ayuda para analizar y generar soluciones a los problemas de una institución. Permite observar aspectos que no se habían tenido en cuenta para mejorar los procesos, métodos y tecnologías, y sirve para generar ideas y dinamizar el proceso de cambio en diferentes niveles de las empresas, de manera que estas innoven en sus procesos internos, sus productos y sus servicios. La innovación requiere una idea inteligente y creativa, pero también una estrategia para desarrollarla y hacerla realidad. La innovación sin método no es posible, pues necesita de una "forma" para alcanzar un estado de innovación. Esta forma está compuesta por acciones planeadas 
deliberadamente, ejecutadas, ajustadas y evaluadas (Castro y Fernández, 2013).

Así, un proceso creativo puede estimularse por medio de diferentes metodologías reconocidas y muy utilizadas en el ámbito académico e industrial: brainstorming, brainwriting, mapas mentales, scamper, PNI (positivo, negativo, interesante), analogías, teoría para resolver problemas de inventiva, design thinking, do it, entre otras. Cuando una idea es perfilada mediante técnicas, herramientas y recursos puede transformarse en nuevos productos, procesos, servicios y negocios.

Una idea perfilada en el marco de la gestión de innovación puede ser el insumo de un instrumento para articular los procesos de investigación con el sector productivo (Barboza, 2008) y, por lo tanto, generar vínculos eficientes que faciliten la integración de intereses y responsabilidades en aspectos operativos, de control y de toma de decisiones (Turriago, 2002).

\section{Conclusiones}

Las instituciones de educación superior están llamadas a convertirse en los centros de $\mathrm{I}+\mathrm{D}+\mathrm{i}$ que dan respuestas a las complejidades del mundo contemporáneo, realizan aportes significativos para el desarrollo en términos de diseño y desarrollo de proyectos productivos, y generan impacto económico, social, ambiental, político y académico. La innovación no depende necesariamente de la tecnología, pues se pueden considerar innovaciones económicas, sociales, tecnológicas, organizativas, estratégicas, etc., que se pueden originar desde la formación, la investigación o las distintas áreas de una institución.

En este sentido, dada la necesidad de que el conocimiento generado, aprendido y enseñado sea útil en el planteamiento de soluciones innovadoras, es aquí donde la gestión del conocimiento institucional debe 
definir la pertinencia temática para atender a las verdaderas necesidades de la región.

La capacidad de innovar en las instituciones de educación superior constituye un recurso más, equivalente a sus capacidades financieras, académicas y productivas. Por tanto, la gestión de la innovación debe ser un proceso permanente, natural y orgánico en la estructura administrativa y formativa de estas instituciones. La producción intelectual, las aplicaciones, los prototipos, los desarrollos materializados y el capital humano formado son elementos que pueden evidenciar procesos de calidad y contribución al Sistema Nacional de Ciencia, Tecnología e Innovación.

Desde esta perspectiva, la innovación debe ser omnidireccional para desarrollar, fomentar y garantizar que se articulen todos los actores y las áreas de la institución. De esta forma, se logra el mejoramiento constante de procesos académicos, investigativos, directivos y administrativos.

\section{Referencias}

Ahmed, P., Shepherd, C., Garza, L. y Garza, C. (2012). Administración de la innovación. Pearson Educación.

Albornoz, M. y López, J. (2010). Ciencia, tecnología y universidad en Iberoamérica. Eudeba.

Angel, R. (2006). Putting an innovation culture into practice. Ivey Bussines Journal. https://iveybusinessjournal.com/publication/putting-an-innovation-culture-intopractice/

Aponte, G. M. (2016). Gestión de la innovación tecnológica mediante el análisis de la información de patentes. Negotium, 33(11), 42-68.

http://ojs.revistanegotium.org.ve/index.php/negotium/article/view/130

Banegas, J. (1999). La cultura de la innovación como reto. Madrid, (2), 14-19. 
Barboza, J. (2008). Gestión de innovación tecnológica universitaria para la transferencia de conocimiento hacia les redes de innovación productiva [Tesis de doctorado]. Universidad Rafael Belloso Chacín.

Benavides, O. (2004). La innovación tecnológica desde una perspectiva evolutiva. Cuadernos de Economía, 23(41), 49-70. https://revistas.unal.edu.co/index.php/ceconomia/article/view/9092

Castro, E. y Fernández, I. (2013). El significado de innovar. CSIC; Catarata.

Cornejo, M. y Muñoz, E. (2009). Percepción de la innovación: cultura de la innovación y capacidad innovadora. Pensamiento Latinoamericano.

Dávila, T., Epstein, M. y Shelton, R. (2006). La innovación que sífunciona. Cómo gestionarla, medirla y obtener beneficio de ella. Ediciones Deusto.

De Bono, E. (1998). Pensamiento lateral. Manual de creatividad. Ediciones Paidós Ibérica.

Drucker, P. (2002). The discipline of innovation. Harvard Business Review. https://hbr.org/2002/08/the-discipline-of-innovation

García, C. (1999). Innovación y cultura: la relevancia de la confianza.

Goldenberg, J., Horowitz, R., Amnon, L. y Mazursky, D. (2003). Cómo dar en el clavo de la innovación. Harvard Business Review América Latina. https://innovationexperience.sitios.udd.cl/files/2014/o7/Art-HBR-Spanish.pdf

Ministerio de Ciencia, Tecnología e Innovación. (2020). Sobre Minciencias. https://minciencias.gov.co/ministerio/sobre-minciencias

Organización para la Cooperación y el Desarrollo y Oficina Estadística de la Unión Europea. (2007). Manual de Oslo. Guía para la recogida e interpretación de datos de innovación (3. ${ }^{\mathrm{a}}$ ed.). Tragsa. https://doi.org/10.1787/9789264065659-es

Ospina, S. (1993). Gestión, política pública y desarrollo social: hacia la profesionalización de la gestión pública. Gestión y Política Pública, 2(1), 35-56.

Rodríguez, M. (1993). Manual de creatividad. Los procesos psíquicos y el desarrollo. Trillas.

Turriago, H. (2002). Gerencia de la innovación tecnológica. Editorial Alfaomega. 
Verworn, B., Lütje, C. y Hertatt, C. (2000). Innovationsmanagement in kleinen and mittleren Unternehmen [Working Paper n. ${ }^{\circ}$ 7]. Institute of Technology and Innovation Management, Hamburg University of Technology.

SIGNOS, Investigación en Sistemas de Gestión

ISSN: 2145-1389 | e-ISSN: 2463-1140 | DOI: https://doi.org/10.15332/24631140

Vol. 13 N. ${ }^{0} 1$ | enero-junio de 2021 